\title{
Structural Relaxation Dynamics and Annealing Effects of Sodium Silicate Glass
}

\author{
Mohamed Naji, ${ }^{\dagger, \S}$ Francesco Piazza, ${ }^{\ddagger, \S}$ Guillaume Guimbretière, ${ }^{\dagger, \S}$ Aurélien Canizarès, ${ }^{\dagger, \S}$ \\ and Yann Vaills $* \dagger, \S$ \\ ${ }^{\dagger}$ CNRS, UPR 3079 CEMHTI, 1D avenue de la Recherche Scientifique, 45071 Orléans, France \\ ${ }^{\ddagger}$ CNRS, UPR 4301 Centre de Biophysique Moleculaire, Rue Charles Sadron, 45071 Orléans, France \\ ${ }^{\S}$ Université d'Orléans, Faculté des Sciences, Avenue du Parc Floral, BP 6749, 45067 Orléans Cedex 2, France
}

ABSTRACT: Here we report high-precision measurements of structural relaxation dynamics in the glass transition range at the intermediate and short length scale for a strong sodium silicate glass during long annealing times. We evidence for the first time the heterogeneous dynamics at the intermediate range order by probing the acoustic longitudinal frequency in the $\mathrm{GHz}$ region by Brillouin light scattering spectroscopy. Or, from in-situ Raman measurements, we show that relaxation is indeed homogeneous at the interatomic length scale. Our results show that the dynamics at the intermediate range order contains two distinct relaxation time scales, a fast and a slow component, differing by about a 10 -fold factor below $T_{g}$ and approaching to one another past the glass transition. The slow

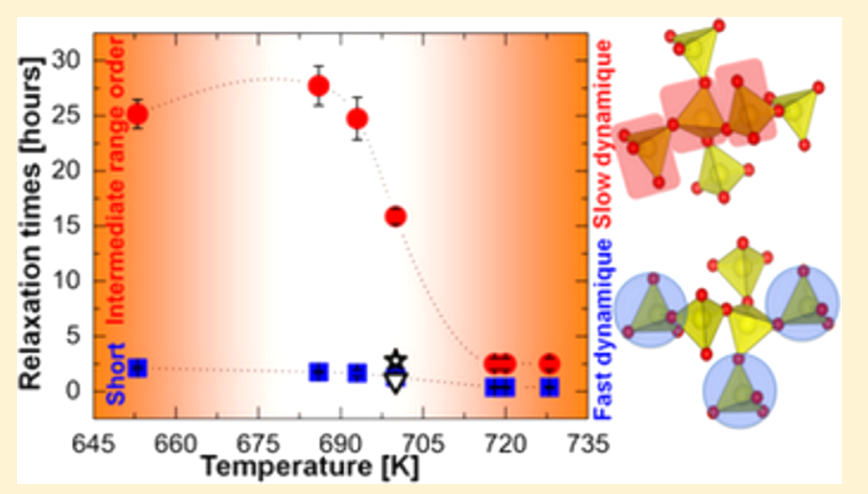
relaxation time agrees with the shear relaxation time, proving that $\mathrm{Si}-\mathrm{O}$ bond breaking constitutes the primary control of structural relaxation at the intermediate range order.

\section{INTRODUCTION}

Intense studies of glass-forming materials have unfolded a comprehensive picture of the dynamical slowing-down during supercooling. ${ }^{1-14}$ For $\mathrm{SiO}_{2}$, often considered as the prototypical strong glass-former, a picture of simple activated dynamics is reasonable, with an almost constant activation energy of about $180 \mathrm{kcal} / \mathrm{mol} .{ }^{15}$ This indicates that the same underlying mechanism of $\mathrm{Si}-\mathrm{O}$ bond breaking and reformation is at work throughout the entire process. ${ }^{16}$ However, in other materials, such as fragile glass-formers, this simple picture fails because of the non-Arrhenius temperature dependence of dynamics. Mechanisms of structural relaxation often assume that groups of molecules rearrange in a cooperative manner. ${ }^{17-19}$ Furthermore, it has been postulated that relaxation dynamics in glasses and supercooled liquids is spatially heterogeneous; that is, the dynamics of group of molecules in one region can be orders of magnitude faster than that of others in another region only a few nanometers away. ${ }^{8,9,12,20}$ Thus, the existence of slow and fast dynamics in the glassy state should also result in annealing rates for these different regions. ${ }^{20}$

In other strong glass formers such as alkali silicate, structural relaxation studies are seldom reported in the literature. Most experiments have traditionally been carried out with dilatometry, enthalpy relaxation, and other methods that probe the macroscopic mechanical or thermal properties of theses glasses. ${ }^{21,22}$ In the vicinity of $T_{g}$, relaxation times determined from these experiments have been shown to be identical to the shear relaxation times obtained from viscosity, ${ }^{21}$ which led to the conclusion that, at least at the probed length scale, the underlying relaxation mechanism is the same, and Si$\mathrm{O}$ bonds are being broken during the relaxation process.

However, most of these studies focused only on empirical or phenomenological characterizations of relaxation dynamics, whereas a complete description of structural relaxation phenomena in these structural heterogeneous systems requires knowledge of dynamics at the atomic and mesoscopic length scale as well.

In this paper, we report direct observation of structural relaxation dynamics and annealing effects over different length scales in the strong glass former sodium silicate. Thus, by coupling information provided by two optical techniques, Brillouin and Raman light scattering spectroscopies, Brillouin light scattering (BLS) spectroscopy provided information over the length scale on the order of the relevant acoustic wavelength while Raman spectroscopy provided information about structural relaxation and annealing dynamics on the molecular length scale. We give evidence of heterogeneous dynamics near the glass transition at the intermediate range order, obtained by probing the propagation of acoustic waves in the $\mathrm{GHz}$ region through BLS spectroscopy during annealing. In-situ Raman spectroscopy measurements further reveal that

Received: January 31, 2013

Revised: March 12, 2013

Published: April 10, 2013 


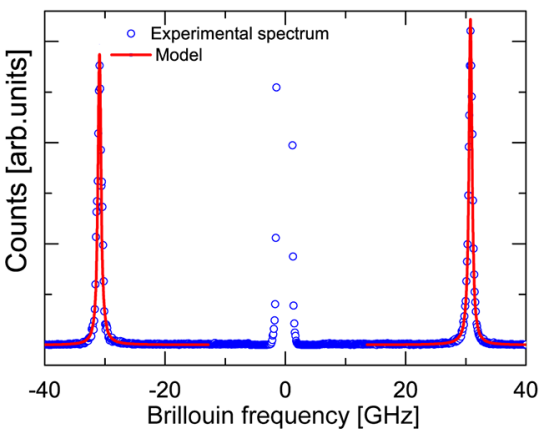

(a)

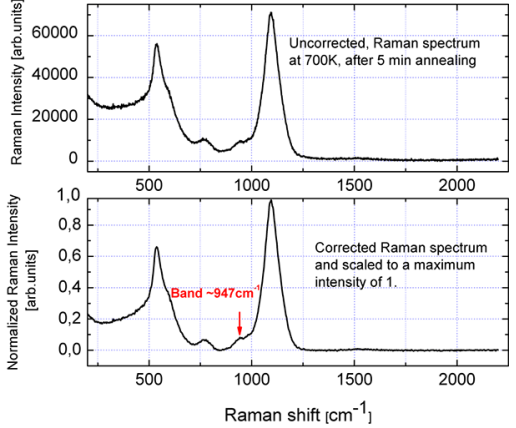

(b)

Figure 1. (a) Representative Brillouin light scattering (BLS) spectrum of $\left(\mathrm{Na}_{2} \mathrm{O}\right)_{0.27}\left(\mathrm{SiO}_{2}\right)_{0.73}$ at room temperature (open circles) together with the best-fit line-shape. (b) Upper panel: Raman spectrum of $\left(\mathrm{Na}_{2} \mathrm{O}\right)_{0.27}\left(\mathrm{SiO}_{2}\right)_{0.73}$ at $700 \mathrm{~K}$ after 5 min annealing. Lower panel: corrected spectrum (background removed and scaled to a maximum intensity of 1 ). The vertical arrow shows the maximum of the band corresponding to the $\mathrm{Q}^{2}$ species.

the relaxation dynamics is indeed homogeneous at the shortrange order.

\section{EXPERIMENTAL SECTION}

Glass Synthesis. The glass chosen for this study is $\left(\mathrm{Na}_{2} \mathrm{O}\right)_{0.27}\left(\mathrm{SiO}_{2}\right)_{0.73}$. The inclusion of $\mathrm{Na}^{+}$ions in the $\mathrm{SiO}_{2}$ network does not induce a remarkable change of the fragility parameter $(m \approx 37)^{22}$ with respect to pure $v-\mathrm{SiO}_{2}(m \approx 20)$, and it shows a strong shift from the decomposition dome. ${ }^{23}$ Ingots of glass samples were prepared by melt of $\mathrm{SiO}_{2}$ (99.9\%) and $\mathrm{Na}_{2} \mathrm{CO}_{3}(99.9 \%)$ in a $\mathrm{Pt}-\mathrm{Rh}$ crucible at about $1900 \mathrm{~K}$ for 4 $\mathrm{h}$ (to eliminate all gas bubbles from the melt and obtain homogeneity) and then poured into a stainless-steel mold kept at room temperature. The as-quenched ingots of glass were cut by a diamond saw into $2 \times 2 \times 1 \mathrm{~mm}^{3}$ samples and optically polished.

Measurements and Experimental Procedures. Brillouin spectra were recorded in a backscattering geometry. The incident light was produced by a single mode $\mathrm{Ar}^{+}$laser operating at $514.5 \mathrm{~nm}$. The spectra were recorded with a sixpass tandem Fabry-Perot interferometer (JRS). ${ }^{24}$ Typical Brillouin spectra in backscattering geometry consist of an elastic peak (Rayleigh line) plus a Brillouin doublet. In order to extract the frequency shifts, each spectrum was adjusted to an elastic line plus a standard damped harmonic oscillator response (Dho), convoluted with the separately measured instrumental response. A typical Brillouin spectrum and the corresponding fitting model are shown in Figure 1a.

In the first set of experiments, the Brillouin spectra were recorded from room temperature up to $\sim 780 \mathrm{~K}\left(T_{g}+\sim 70 \mathrm{~K}\right)$ at a heating rate of $5 \mathrm{~K} / \mathrm{min}$ for an as-quenched $(\mathrm{aQ})$ glass sample. The $(\mathrm{aQ})$ glass was kept at this temperature for $2 \mathrm{~h}$ and then slowly cooled down to room temperature. The resulting glass sample, referred to as annealed sample (A), was then reheated at a rate of $5 \mathrm{~K} / \mathrm{min}$ up to $780 \mathrm{~K}$, and the Brillouin spectra were registered again.

In the second set of Brillouin light scattering experiments, the spectra were recorded isothermally (i.e., at a fixed temperature) as a function of time (up to $72 \mathrm{~h}$ ) after a fast jump $(50 \mathrm{~K} / \mathrm{min})$ from room temperature to the chosen annealing temperature $(653,686,693,700,718,720$, and 728 $\mathrm{K})$. For each experiment, a new $(\mathrm{aQ}$ ) glass sample was used. Acquisition times of 300 cycles were used $(\sim 3 \mathrm{~min})$ for each spectrum with a waiting time of $3 \mathrm{~s}$ between consecutive spectra. Up to 1000 spectra were recorded for long experiments, thus considerably improving the statistics.
In-situ Raman spectra were recorded with a confocal InVia Reflex Renishaw in backscattering geometry, with the $514 \mathrm{~nm}$ line of an argon laser using a long working distance objective $(\times 20)$. The Raman scattered light was dispersed with a holographic grating with 1800 lines/mm and detected with a charge-coupled device (CCD) camera. All Raman spectra were obtained in the VV polarization configuration (parallel polarizations of incident and scattered light) and with an acquisition time of $300 \mathrm{~s}$ with one accumulation. Raman spectra were registered isothermally for the $(\mathrm{aQ})$ glass, after a fast jump $(50 \mathrm{~K} / \mathrm{min})$ from room temperature into the chosen temperature $(700 \mathrm{~K})$ for $34 \mathrm{~h}$. The temperature was controlled using a TS 1500 Linkam micro-oven device. Figure $1 \mathrm{~b}$ (upper panel) shows a typical Raman spectrum collected at $700 \mathrm{~K}$ after 5 min of annealing. The spectral background was removed by fitting a second order polynomial to the spectral region of $1250-1800 \mathrm{~cm}^{-1}$, where no Raman peaks are present, extrapolating the polynomial to lower frequencies and subtracting it from the spectrum. The spectra were scaled to a maximum intensity of 1 and not corrected by the BoseEinstein factor, as we only compare spectra obtained at constant temperature during annealing (Figure $1 \mathrm{~b}$ ) (lower panel).

\section{RESULTS}

Intermediate Range Order. Figure 2 shows the Brillouin frequency shift $\nu_{B}$ as a function of temperature for the asquenched $(\mathrm{aQ}$, solid circles) and annealed ( $\mathrm{A}$, squares) glass samples, recorded during the first set of experiments (heating rate $5 \mathrm{~K} / \mathrm{min}$ ). The data shows that the longitudinal Brillouin frequency curves $\nu_{B}(T)$ of both glasses present an inflection point appearing at $\sim 710 \mathrm{~K}$ which is the manifestation of the glass-liquid transition at $T_{g}$ at the experimental time scale (characteristic of BLS $10^{-10^{g}} \mathrm{~s}$ ). Moreover, the annealed glass (A) shows a higher Brillouin frequency $\nu_{B}$ than the as-quenched one $(\mathrm{aQ})$ over all the temperature domain below $T_{g}$, showing that annealing causes the $(\mathrm{aQ})$ glass sample to relax to the $(\mathrm{A})$ state, where $\nu_{B}$ is up to about $1.6 \%$ higher than the initial $(\mathrm{aQ})$ state. A close inspection of data reveals a small change of slope of the $(\mathrm{aQ})$ Brillouin frequency curve at temperatures between 660 and $700 \mathrm{~K}$. This corresponds to a temperature range where the characteristic time(s) of the structural relaxation becomes comparable to the holding time set by the slow heating rate $(5$ $\mathrm{K} / \mathrm{min}$ ). Above $T_{g}$, network relaxation becomes faster, and equilibrium is quickly achieved. Hence, no difference has been observed between both glasses (Figure 2). The gradual 


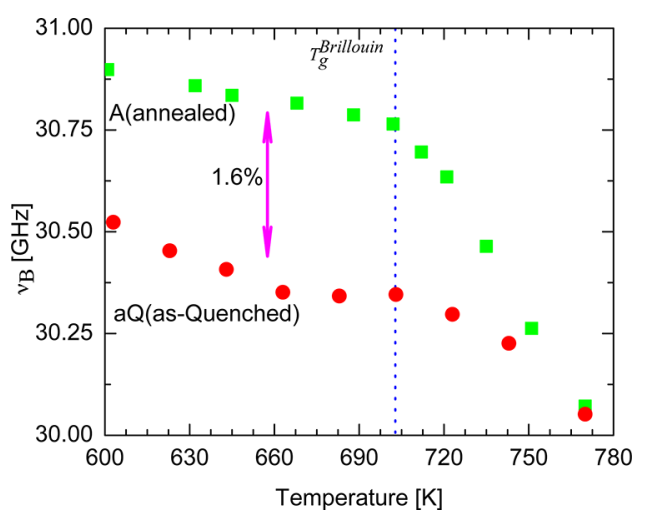

Figure 2. Brillouin frequency shift $\nu_{B}$ of as-quenched (aQ) (solid circles) and annealed (A) (squares) glass samples versus temperature. The vertical dotted line indicates the glass transition $T_{g}$ as estimated by BLS.

decrease of $\nu_{B}$ for both glasses in (aQ) and (A) states upon heating is a blueprint of anharmonic effects observed in such glass system and other oxide glasses in the gigahertz frequency range. ${ }^{25-27}$ Our relaxation experiments consisted in a fast temperature rise $(50 \mathrm{~K} / \mathrm{min})$ from room temperature to a given temperature in the glass transition region, followed by an isothermal annealing where we monitor the relaxation dynamics of the glass by BLS as a function of time. Figure 3 displays a

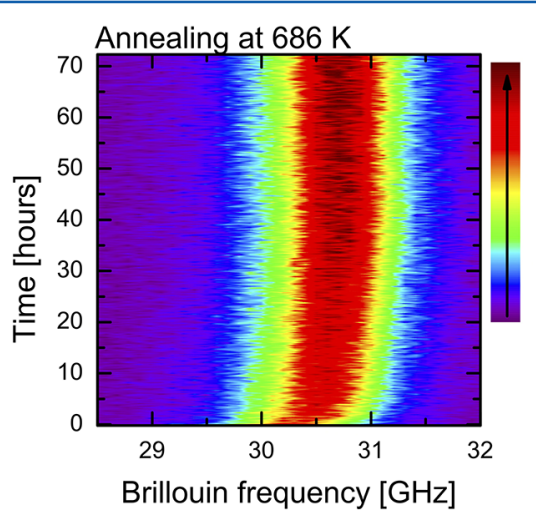

Figure 3. Time-Brillouin frequency contour plot of BLS intensity for the $(\mathrm{aQ})$ sample heated at $686 \mathrm{~K}$. The $x$-axis is the Brillouin frequency $(28.5-32 \mathrm{GHz}$ ), the $y$-axis is the annealing time, and the $z$-axis (color scale) corresponds to BLS intensity.

typical time evolution of the Brillouin spectra in the 28.5-32 $\mathrm{GHz}$ frequency region for an $(\mathrm{aQ})$ glass during annealing at $686 \mathrm{~K}$. As one can see, Brillouin frequency shows an evolution toward higher frequencies until saturation (Figure 3).

Figure $4 \mathrm{a}$ displays the measured isothermal relaxation dynamics of the Brillouin frequency at four temperatures below $T_{g}$ as a function of the annealing time. The exponential peeling method (illustrated for one representative kinetics in the inset in Figure 4a) shows that the data clearly follow a double-exponential relaxation profile of the type

$$
\nu_{B}(t)=\nu_{B}^{\infty}-w_{1} \mathrm{e}^{-t / \tau_{1}}-w_{2} \mathrm{e}^{-t / \tau_{2}}
$$

where $\nu_{B}^{\infty}$ is the asymptotic frequency, $\tau_{1}$ and $\tau_{2}$ are the relaxation times, and $w_{1}$ and $w_{2}$ describe the corresponding exponential weights. The two time scales $\left(\tau_{1}\right.$ and $\left.\tau_{2}\right)$ obtained from fitting the data with eq 1 appear to be separated by a 10fold factor, as shown in Figure 5, while the associated weights $\left(w_{1}\right.$ and $\left.w_{2}\right)$ are roughly the same and appear rather temperature-independent (see caption of Figure $4 a$ ). We remark that, for temperatures below $T_{g}$, a stretched-exponential curve of the type $\nu_{B}(t)=\nu_{B}^{\infty}-w \exp \left[-(t / \tau)^{\beta}\right]$, with $0 \leq \beta \leq 1$, could not account for the measured relaxation over the whole temperature range, as it systematically yielded overstretched relaxations, with best-fit values of $\beta$ as low as 0.1 . This typically occurs when few and well distinct relaxation time scales are present (as in our case), as opposed to the case of multiple and closely spaced time scales, confirming the soundness of our theoretical approach. The error bars $\Delta \tau_{1}$ and $\Delta \tau_{2}$, as shown in Figure 5, have been estimated as follows. First, we generated artificial relaxation data with fixed decay times $\tau_{1}$ and $\tau_{2}$, with $\tau_{2}$ $=10 \tau_{1}$. To the double-exponential relaxation we then added Gaussian noise matching the experimentally observed one, estimated from the standard deviation of the residuals to the fits. Then a calibration table was built by fitting increasingly long portions of the surrogate data and recording the statistical errors on the best-fit values of the time constants as a function of the length of the fitted time series. Fits were performed with the nonlinear least-squares Marquardt-Levenberg algorithm. Accordingly, statistical uncertainties in the fitting parameters were obtained from the diagonal elements of the inverse of the $\chi^{2}$ Hessian matrix. The calibration curves constructed through this procedure have been subsequently used to estimate $\Delta \tau_{1}$ and $\Delta \tau_{2}$ by matching the actual length of the measured relaxation data sets within the calibration table.

Things appear to change as the glass transition is reached. As expected, in this temperature region the evolution toward equilibrium is much faster (Figure $4 \mathrm{~b}$ ). We found that the more reliable way to estimate $\tau_{1}$ and $\tau_{2}$ was to recognize, in agreement with the data, that the measured kinetics do not vary appreciably over the $10 \mathrm{~K}$ measured temperature span. Consequently, we can reduce the uncertainties associated with the fitting procedure by fitting the average relaxation curve. In Figure 5 we report the corresponding best-fit values $\tau_{1}$ $=22.4 \mathrm{~min}$ and $\tau_{2}=2.5 \mathrm{~h}$. The error bars match in this case the observed variation of $\tau_{1}$ and $\tau_{2}$ obtained by fitting the three kinetics (Figure 5). The relaxation curves above $T_{g}$ are shown in Figure $4 b$, together with the double-exponential fits performed by keeping the two time scales fixed at the average relaxation times and letting the other parameters float. It is interesting to observe that the measured kinetics above the glass transition are coherently equally well fitted by (i) two closely spaced exponentials and (ii) a single stretched exponential of the type (eq 2 ) with $\beta=1 / 2$.

$$
\nu_{B}(t)=\nu_{B}^{\infty}-w \exp \left[-(t / \tau)^{\beta}\right]
$$

Interestingly, the fit with the stretched exponential to the average relaxation gives $\tau=52 \mathrm{~min} \approx\left(\tau_{1} \tau_{2}\right)^{1 / 2}$ and $w \approx 0.38$. Physically, the emerging picture is that the two relaxation processes associated with well separated time scales below $T_{g}$ are now merging into a rather temperature-insensitive mechanism. Thus, the observed heterogeneity is strongly attenuated above $T_{g}$, and its characteristic length scale seems to rapidly decrease upon increasing temperature.

Short-Range Order. The question that should be addressed is whether the heterogeneous dynamic behavior is also present in the short-range order. To answer this question, we additionally carried out an annealing experiment at $700 \mathrm{~K}$ in the $(\mathrm{aQ})$ glass following the same experimental procedure $(50$ $\mathrm{K} / \mathrm{min}$ ) and probing the interatomic range order by microRaman spectroscopy. 


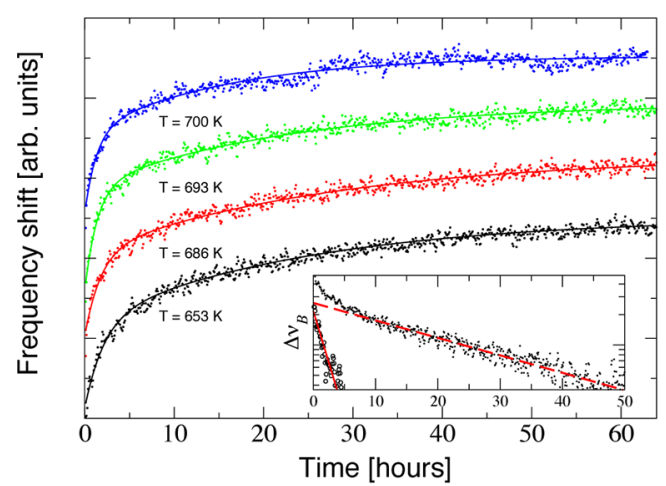

(a)

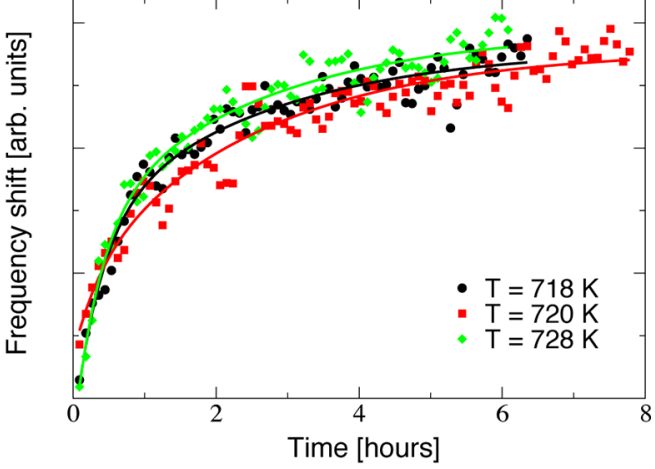

(b)

Figure 4. (a) Relaxation curves of $(\mathrm{aQ})$ samples as a function of the annealing time after a fast temperature step ( $50 \mathrm{~K} / \mathrm{min})$ from room temperature to $T<T_{g}$ (curves have been shifted vertically for clarity). Filled points mark the data, while solid lines are fits to eq 1 . Best-fit values of the weights $w_{1}, w_{2}$ are as follows: $0.21,0.22(T=653 \mathrm{~K}), 0.20,0.24(T=686 \mathrm{~K}), 0.22,0.20(T=693 \mathrm{~K}), 0.20,0.20(T=700 \mathrm{~K})$. The inset illustrates the exponential peeling decomposition for the kinetic at $T=653 \mathrm{~K}$. Here $\Delta \nu_{B}(t)=\nu_{B}^{\infty}-\nu_{B}(t)$ is plotted on a semilogarithmic scale. The dashed line is the slow exponential law $f_{2}(t)=w_{2} \mathrm{e}^{-t / \tau_{2}}$ (see again eq 1 ). Empty circles reveal the fast relaxation, obtained as $\Delta \nu_{B}(t)-f_{2}(t)$. The solid line is the corresponding fit to the exponential $w_{1} \mathrm{e}^{-t / \tau_{1}}$. (b) Relaxation curves of $(\mathrm{aQ})$ samples as a function of the annealing time after a fast temperature step ( $50 \mathrm{~K} / \mathrm{min}$ ) from room temperature to $T>T_{\text {g. }}$. Filled points mark the data (evolution of the Brillouin shift). The solid lines are fits to eq 1 with fixed relaxation times $\tau_{1}=22.4 \mathrm{~min}$ and $\tau_{2}=2.5 \mathrm{~h}$ (average relaxation times of the three kinetics). Best-fit values of the weights $w_{1}, w_{2}$ are as follows: 0.16 , $0.14(T=718 \mathrm{~K}), 0.07,0.18(T=720 \mathrm{~K}), 0.17,0.16(T=728 \mathrm{~K})$.

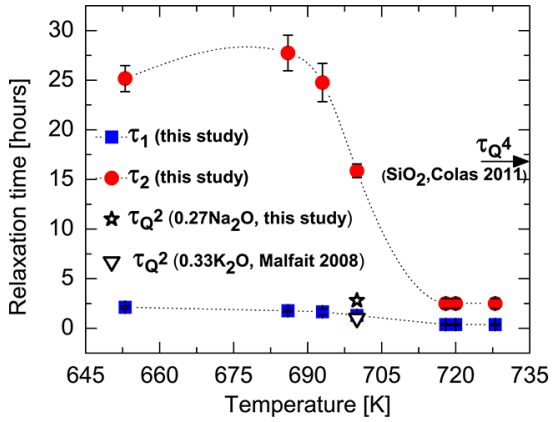

Figure 5. Best-fit values of the relaxation times $\tau_{1}$ and $\tau_{2}$ measured by BLS for the structural relaxation of $\left(\mathrm{Na}_{2} \mathrm{O}\right)_{0.27}\left(\mathrm{SiO}_{2}\right)_{0.73}$ as a function of the annealing temperatures. Above $T_{g}$ we report the average relaxation times for the three kinetics at $T=718,720$, and $728 \mathrm{~K}$. The relaxation time of the $\mathrm{Q}^{2}$ species measured by Raman spectroscopy for the $(\mathrm{aQ})$ sample is displayed as an open star, while the one obtained from the literature ${ }^{28}$ for $\left(\mathrm{K}_{2} \mathrm{O}\right)_{0.33}\left(\mathrm{SiO}_{2}\right)_{0.67}$ is shown as an open triangle. The right-pointing-arrow indicates the measured relaxation time ${ }^{29}$ for $\mathrm{SiO}_{2}$ at $T=1373 \mathrm{~K}$. Dotted lines are guides to the eye.

Figure $1 \mathrm{~b}$ shows a typical Raman spectra recorded after 5 min of annealing at $700 \mathrm{~K}$. The high frequency region between 850 and $1200 \mathrm{~cm}^{-1}$ reports structural information about shortrange order. ${ }^{30-32}$ We have used a nonlinear least-squares fitting routine to fit this spectral region to Gaussian components. We find a good fit, with four Gaussian components centered at 947, 1040,1100 , and $1144 \mathrm{~cm}^{-1}$. All band parameters (frequency, half-width and intensity) are independent variables in the fitting routine. Our spectrum decovolution is comparable to those reported in previous studies for a very broad range of highconcentration alkali silicate glasses. ${ }^{31-34} \mathrm{~A}$ typical fit is shown in Figure 6a. In $\left(\mathrm{Na}_{2} \mathrm{O}\right)_{0.27}\left(\mathrm{SiO}_{2}\right)_{0.73}$ glass, previous Raman and NMR data reveal the coexistence of $\mathrm{Q}^{n}$ structural units, where $n$ is the number of bridging oxygens cross-linking the silica tetrahedra and $4-n$ is the number of nonbridging oxygens (NBO).$^{31-35}$ The bands near 947 and $1100 \mathrm{~cm}^{-1}$ are assigned to $\mathrm{Si}-\mathrm{O}^{-}$stretching motion in respectively $\mathrm{Q}^{2}$ and $\mathrm{Q}^{3}$ structural units. ${ }^{30,34,36,37}$ The band centered at $1144 \mathrm{~cm}^{-1}$ is
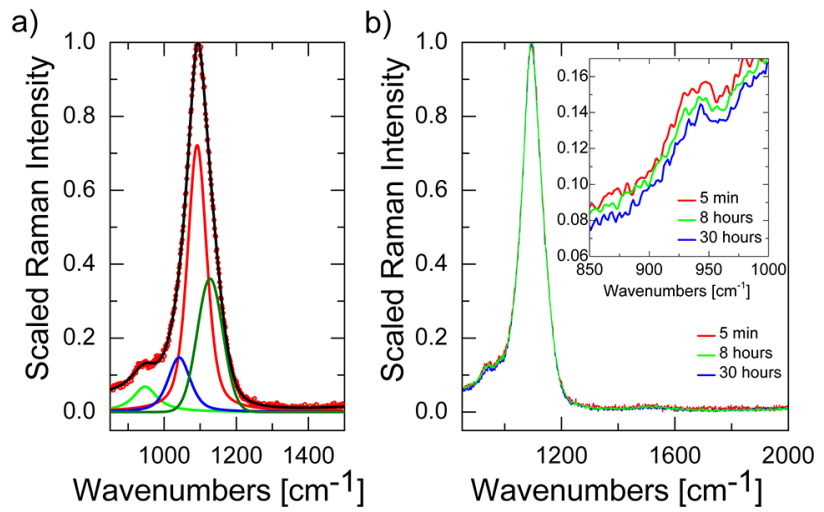

Figure 6. (a) Example of typical curve-fitted, high frequency region of $\left(\mathrm{Na}_{2} \mathrm{O}\right)_{0.27}\left(\mathrm{SiO}_{2}\right)_{0.73}$ (taken after $5 \mathrm{~min}$ of annealing at $700 \mathrm{~K}$ ). (b) Selected Raman spectra taken after different annealing times. The intensities are calculated relative to the greatest intensity within each spectrum. The inset shows the evolution of the peak maxima at the $947 \mathrm{~cm}^{-1}$ band, corresponding to $\mathrm{Q}^{2}$ species.

assigned to $\mathrm{Si}-\mathrm{O}$ stretching motion in fully polymerized structural units $\left(Q^{4}\right){ }^{30,34,36,37}$ The assignment of the 1040 $\mathrm{cm}^{-1}$ band is somewhat controversial. On the basis of comparison of Raman spectra of vitreous $\mathrm{SiO}_{2}$ and other silica-poor compositions, Lasaga ${ }^{38}$ and Mysen et al. ${ }^{39,40}$ suggested that this band could be assigned to a $\mathrm{Si}-\mathrm{O}$ stretching vibration arising from bridging oxygen but not necessarily residing in fully polymerized structural units, whereas other authors assigned it to a vibration associated with alkali cations. ${ }^{41}$ Even though the assignment of this band is problematic, its presence is definitely required from the viewpoint of deconvolution with symmetric Gaussian lines. As shown in Figure 6b, changes of the vibrational signature as a function of time during annealing are very smooth. Careful examination of this data shows that changes in the spectra do indeed occur and affect the intensity of the peak at $947 \mathrm{~cm}^{-1}$ (assigned to the stretching vibration of $\mathrm{Q}^{2}$ structural units), relative to the peak at $1100 \mathrm{~cm}^{-1}$ (inset in Figure 6b). The 


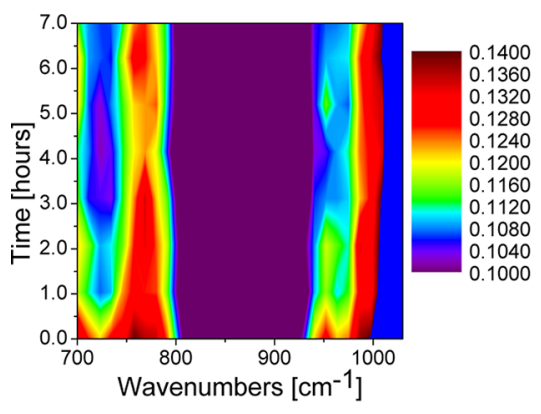

(a)

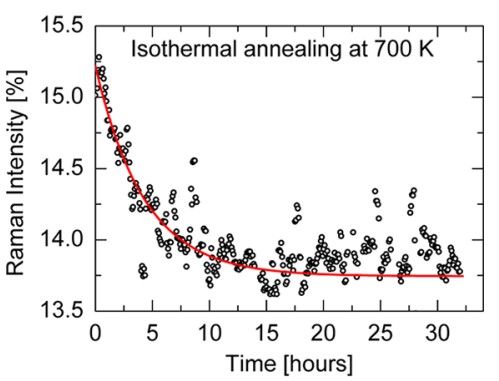

(b)

Figure 7. (a) Time-wavenumber contour plot of Raman intensity for the $(\mathrm{aQ})$ sample heated at $700 \mathrm{~K}$. The $x$-axis is the Raman wavenumber shift (700-1050 $\mathrm{cm}^{-1}$ ), the $y$-axis is the annealing time (for clarity we show only the first $7 \mathrm{~h}$ of annealing), and the $z$-axis (color scale) corresponds to Raman intensity in the range 0.1-0.14. This plot allows visualization of the decrease of the intensity of the band at $947 \mathrm{~cm}^{-1}$. (b) Intensity of the peak maxima (full circles) of the $947 \mathrm{~cm}^{-1}$ band corresponding to $\mathrm{Q}^{2}$ species as a function of the annealing time. Solid line is an exponential fit using eq 3 , giving a relaxation time $\tau_{Q^{2}} \approx 3 \mathrm{~h}$.

visualization as the intensity map of Figure 7a allows revealing these small but unambiguous changes, owing to the gain of statistics given by the juxtaposition of numerous spectra. The relative Raman intensity of this band as a function of the annealing time displays a simple exponential decay:

$$
I(t)=I^{\infty}+a \exp \left[-\left(t / \tau_{Q^{2}}\right)\right]
$$

where $I^{\infty}$ is the asymptotic intensity, $a$ is the exponential weight, and $\tau_{Q^{2}} \approx 3 \mathrm{~h}$ is the relaxation time (Figure $7 \mathrm{~b}$ ). Compared to the relaxation times obtained from BLS, $\tau_{Q^{2}}$ falls close to the fast BLS relaxation time scale $\tau_{1}$ (open star in Figure 5) and is about 1 order of magnitude faster than the slow relaxation dynamics. The decrease in peak intensity would indicate a reduced abundance of $\mathrm{Q}^{2}$ species as the as-quenched $(\mathrm{aQ})$ glass sample changes configuration upon approaching equilibrium. This change in abundance of $Q^{2}$ units suggests that the disproportionation reaction (eq 4) shifted to the left with annealing.

$$
2 Q^{3} \rightleftharpoons Q^{2}+Q^{4}
$$

However, as the changes are very small and bands assigned to $\mathrm{Q}^{3}$ and $\mathrm{Q}^{4}$ units are overlapped within the high frequency envelope, a quantitative description of this re-equilibration from Raman data requires knowledge of the relationship between the band intensities and species concentrations dependence at different annealing temperatures.

The $\tau_{Q^{2}}$ relaxation time scale is very close to the one reported by Malfait et al. ${ }^{28}$ for $\mathrm{K}_{2} \mathrm{O}-\mathrm{SiO}_{2}$ with 29.4 and $33.3 \% \mathrm{~K}_{2} \mathrm{O}$. Furthermore, the relaxation dynamics of $\mathrm{Q}^{2}$ in both glass systems at the same temperature range $\left(T / T_{g} \approx 0.9\right)$ are both exponential $(\beta=1)$. Thus, structural relaxation in these glass systems at this temperature range is far to be affected by the nature of the oxide modifier $\left(\mathrm{Na}_{2} \mathrm{O}\right.$ or $\left.\mathrm{K}_{2} \mathrm{O}\right)$, as confirmed from electrical conductivity measurements, where the sodium mobility involves much shorter time scales. ${ }^{42,43}$

Information from Raman spectra about structural relaxation in the intermediate range order seems to be difficult for two reasons: (i) the mode ascribed to intertetrahedral $\mathrm{Si}-\mathrm{O}-\mathrm{Si}$ bending motion is highly attenuated for this glass composition, and (ii) several vibrational modes may contribute to the Raman intensity in the spectral region where information on the intermediate range structure can be extracted. However, referring to silica, some of the present authors have shown that structural relaxation involves changes in the interterahedral $\mathrm{Si}-\mathrm{O}-\mathrm{Si}$ angles, ${ }^{29}$ with a relaxation time $\left(\tau_{Q^{4}}\right)$ of about $17 \mathrm{~h}$ at temperatures below $T_{g}\left(T / T_{g} \approx 0.9\right)$. Tomozawa et al. ${ }^{44}$ have reported similar relaxation time $(\sim 15 \mathrm{~h})$ of vibrational features of silica at the interatomic scale using infrared (IR) spectroscopy. Comparing these relaxation time scales to our measured $\tau_{2}$, one can immediately see that these times are in good agreement.

\section{DISCUSSION}

Several relaxation studies have confirmed the heterogeneous relaxation dynamics in the so-called Fragile glass formers, which include polymers, and most molecular glass-formers. ${ }^{1-14}$ There is, however, weak knowledge on the dynamics of the so-called Strong glass formers, such as alkali-silicate glasses. Most of the relaxation experiments performed on alkali-silicate glasses have probed structural relaxation indirectly and have been limited to the interpretation of a macroscopic property as a function of temperature and time. ${ }^{21,22}$ Presently, we have probed the structural relaxation dynamics in a typical sodium silicate glass at different length scales during annealing. We have evidenced the heterogeneous relaxation dynamics at the intermediate length scale with a continuous monitoring of the propagation of the longitudinal acoustic waves, while we have shown from Raman data that relaxation dynamics is indeed homogeneous at the interatomic length scale. At the intermediate length scale, we have identified two well distinct relaxation time scales, involving a slow and a fast process. At the short length scale, the determined relaxation time attributed to the stretching vibration of the $Q^{2}$ species is of the same order as the fast relaxation dynamics identified at the intermediate length scale.

Decoupling the observed relaxation from the very common Johari-Goldstein (JG) $\beta$ relaxation is of great interest. ${ }^{45,46}$ However, in strong glass-formers (such as sodium silicate), the dielectric loss spectrum lacks a peak or shoulder on the high frequency side, and it has been found to be impossible to distinguish it from the primary $(\alpha)$ relaxation. ${ }^{47-49}$ Many explanations have been suggested by different authors. Some authors assume that JG relaxation does not exist for these glass formers at all, ${ }^{50,51}$ while others attribute the lack of the JG peak to the fact that this peak is hidden by the $\alpha$ peak in the dielectric loss curves. ${ }^{52}$ In hyperquenched $\mathrm{GeO}_{2}(m \sim 17-20)$, $\mathrm{W}$. $\mathrm{Hu}$ et al. reported a very sophisticated experimental relaxation study and found that JG relaxation is hidden by the primary $\alpha$ relaxation. ${ }^{52}$ In the frame of this description, we suggest that in our established relaxation all the secondary relaxation units might contribute to the primary $\alpha$ relaxation. In 
order to get a clear idea about the mechanism of structural relaxation in this glass-forming liquid, it is instructive to compare our relaxation times $\tau_{1}$ and $\tau_{2}$ to the shear relaxation time $\tau_{\text {shear }}$ obtained from shear viscosity $\eta$ data ${ }^{53}$ using the Maxwell equation $\tau_{\text {shear }}=\eta / G_{\infty}$. $G_{\infty}$ is the shear modulus at infinite frequency, which is considered to be constant $\left(G_{\infty} \approx 10\right.$ $\mathrm{GPa})$ for silicate melts. ${ }^{54}$ It can be seen from Figure 8 that the slow relaxation time scale $\tau_{2}$ agrees with $\tau_{\text {shear }}$ in the glass transition region, or the fast one $\tau_{1}$ is by all means smaller than $\tau_{\text {shear }}$ below and across $T_{g}$.

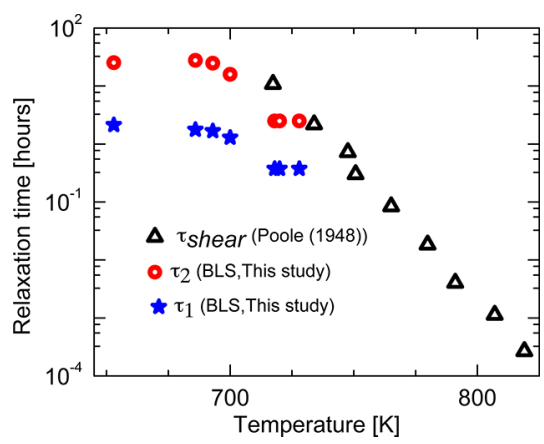

Figure 8. Temperature dependence of the shear relaxation time $\tau_{\text {shear }}$ calculated from shear viscosity data as found in the literature. ${ }^{53}$ The $\tau_{1}$ (blue stars) and $\tau_{2}$ (red open circles) relaxation times calculated from BLS are also plotted.

At this point, two hypotheses for the origin of the observed heterogeneous relaxation dynamics could be drawn. The first attributes this process to a thermal relaxation of the glass sample in response to the fast temperature rise, followed by a very slow exponential structural relaxation process. However, such a scenario is far from possible, since the time to reach thermal equilibrium should be less than $1 \mathrm{~h}$, and the structural relaxation process (slow relaxation in our case) is known to be nonexponential, with a $\beta=0.68$ for this glass former (which is not our case). The second plausible hypothesis could be that the observed heterogeneous dynamics is a direct consequence of the nonexponential character of structural relaxation near $T_{g}$ resulting from dynamic and spatial heterogeneities in the glass sample.

From a structural point of view, the modified random network (MRN) model offers a fruitful way to simplify complexities in the structure of alkali silicate glasses at the intermediate range order. ${ }^{55,56}$ Experimental findings corroborated with molecular dynamics (MD) simulations ${ }^{57-64}$ reveal new intermediate-range order and microstructure characterized by the existence of channels (i.e., percolation pathways of alkali ions that can be mapped out by connection of NBO together) in a MRN. As a consequence, one can imagine at least two different set of environment, namely silicate tetrahedra close to the pathways of $\mathrm{Na}$ ions (in the boundaries of silicate $\mathrm{Si}-\mathrm{O}$ chain) and those within a silicate $\mathrm{Si}-\mathrm{O}$ chain (intervening $\mathrm{Si}-$ $\mathrm{O}-\mathrm{Si}$ bond angles). The agreement between the fast relaxation time observed by Brillouin spectroscopy $\tau_{1}$ and the relaxation time $\tau_{Q^{2}}$ of the band attributed to the stretching vibration of $Q^{2}$ obtained from Raman spectroscopy could correspond to some local motions of $\mathrm{SiO}_{4}$ tetrahedra located at the boundary of the silicate chain (close to pathways of $\mathrm{Na}$ ions). On the other hand, the agreement between the slow relaxation time observed by Brillouin spectroscopy $\tau_{2}, \tau_{Q^{4}}$ (Raman spectroscopy), and $\tau_{\text {shear }}$ (Figure 5 ) strongly suggests that $\mathrm{Si}-\mathrm{O}$ bonds are being broken during the observed relaxation, affecting the $\mathrm{Si}-\mathrm{O}-\mathrm{Si}$ bond angles over long distances. On the basis of this approach, one can argue that the observed dynamics might correspond to the following sequence of events. (i) The system relaxes through a nonexponential process governed by relaxation of the individual spatial regions at a short length scale by a local motion of silicate tetrahedra at the boundaries of $\mathrm{Si}-\mathrm{O}$ chains. (ii) Then this phenomenon is followed by a slow relaxation over long distances: intervening $\mathrm{Si}-\mathrm{O}-\mathrm{Si}$ bond angles where relaxation is primarily controlled by the rate of $\mathrm{Si}-\mathrm{O}$ bond breaking. One has to notice that the experimentally determined heterogeneous dynamics could not be described in the framework of the Tool-Narayanaswamy-Moynihan (TNM) model, as is usually done in annealing experiments. Such a model appears inadequate to describe the heterogeneous relaxation dynamics emerging from our experiments, suggesting that a single fictive temperature is insufficient for pinpointing the structural state in nonequilibrium conditions. ${ }^{65,66}$

\section{CONCLUSION}

In conclusion, relaxation dynamics involving multiple time scales have long been considered a hallmark of systems near $T_{g}$. No experiments on binary oxide glasses had yet evidenced the heterogeneous dynamics of the structural relaxation of these systems. The in-situ Brillouin and Raman measurements depicted in this work evidence a novel and very promising way to follow the relaxation dynamics of glasses over various distances. The central result of our study is a direct experimental account of heterogeneous dynamics near the glass transition in a strong glass former sodium silicate at the intermediate range order: Two well distinct relaxation channels were identified, involving a slow and a fast process. At the short-range order, Raman spectroscopic measurements in $\left(\mathrm{Na}_{2} \mathrm{O}\right)_{0.27}\left(\mathrm{SiO}_{2}\right)_{0.73}$ revealed a homogeneous relaxation near $T_{g}$. The fast relaxation time scale determined from BLS measurements is of the same order as relaxation dynamics corresponding to local rearrangements of $Q^{2}$ species, while the slow relaxation dynamics occurs at the same time scale as the $\mathrm{Si}-\mathrm{O}-\mathrm{Si}$ bending vibration observed in silica around the glass transition. Additionally, an Adam-Gibbs reading of the agreement between the slow relaxation time scale and the shear relaxation time in the glass transition region suggests that $\mathrm{Si}-\mathrm{O}$ bond breaking is at the origin of the observed cooperative structural relaxation at long distances.

\section{AUTHOR INFORMATION}

\section{Corresponding Author}

*E-mail: vaills@cnrs-orleans.fr.

\section{Notes}

The authors declare no competing financial interest.

\section{ACKNOWLEDGMENTS}

We gratefully thank Drs. Patrick Simon, Domingos de souza Menses, and Pierre Florian for their fruitful discussions. We thank Dr. Filippo Scarponi (Ghost-group at Perugia University) for providing BLS software adapted for time-acquisition. This project was supported by the French ministry of research and education. 


\section{REFERENCES}

(1) Schmidt-Rohr, K.; Spiess, H. W. Nature of Nonexponential Loss of Correlation above the Glass Transition Investigated by Multidimensional NMR. Phys. Rev. Lett. 1991, 66, 3020-3023.

(2) Cicerone, M.; Ediger, M. Relaxation of Spatially Heterogeneous Dynamic Domains in Supercooled ortho-Terphenyl. J. Chem. Phys. 1995, 103, 5684-5692.

(3) Cicerone, M.; Ediger, M. Enhanced Translation of Probe Molecules in Supercooled o-Terphenyl: Signature of Spatially Heterogeneous Dynamics? J. Chem. Phys. 1996, 104, 7210-7218.

(4) Perera, D.; Harrowell, P. Measuring Diffusion in Supercooled Liquids: The Effect of Kinetic Inhomogeneities. J. Chem. Phys. 1996, $104,2369$.

(5) Schiener, B.; Böhmer, R.; Loidl, A.; Chamberlin, R. V. Nonresonant Spectral Hole Burning in the Slow Dielectric Response of Supercooled Liquids. Science 1996, 274, 752-754.

(6) Tracht, U.; Wilhelm, M.; Heuer, A.; Feng, H.; Schmidt-Rohr, K.; Spiess, H. W. Length Scale of Dynamic Heterogeneities at the Glass Transition Determined by Multidimensional Nuclear Magnetic Resonance. Phys. Rev. Lett. 1998, 81, 2727-2730.

(7) Donati, C.; Glotzer, S.; Poole, P.; Kob, W.; Plimpton, S. Spatial Correlations of Mobility and Immobility in a Glass-Forming LennardJones Liquid. Phys. Rev. E 1999, 60, 3107.

(8) Ediger, M. Spatially Heterogeneous Dynamics in Supercooled Liquids. Annu. Rev. Phys. Chem. 2000, 51, 99-128.

(9) Vidal Russell, E.; Israeloff, N. E. Direct Observation of Molecular Cooperativity near the Glass Transition. Nature 2000, 408, 695-698.

(10) Weeks, E. R.; Crocker, J. C.; Levitt, A. C.; Schofield, A.; Weitz, D. A. Three-Dimensional Direct Imaging of Structural Relaxation Near the Colloidal Glass Transition. Science 2000, 287, 627-631.

(11) Wang, C.; Ediger, M. Lifetime of Spatially Heterogeneous Dynamic Domains in Polystyrene Melts. J. Chem. Phys. 2000, 112, 6933.

(12) Deschenes, L.; Bout, D. Heterogeneous Dynamics and Domains in Supercooled o-Terphenyl: A Single Molecule Study. J. Phys. Chem. B 2002, 106, 11438-11445.

(13) Paluch, M.; Roland, C. M.; Pawlus, S.; Zioło, J.; Ngai, K. L. Does the Arrhenius Temperature Dependence of the Johari-Goldstein Relaxation Persist above $T_{g}$ ? Phys. Rev. Lett. 2003, 91, 115701.

(14) Capaccioli, S.; Ngai, K. L. Relation between the $\alpha$-Relaxation and Johari-Goldstein $\beta$-Relaxation of a Component in Binary Miscible Mixtures of Glass-Formers. J. Phys. Chem. B 2005, 109, 9727-9735.

(15) Mackenzie, J. D. Viscosity-Temperature Relation for Network Liquids. J. Am. Ceram. Soc. 1961, 44, 598-601.

(16) Debenedetti, P.; Truskett, T.; Lewis, C.; Stillinger, F. Theory of Supercooled Liquids and Glasses: Energy Landscape and Statistical Geometry Perspectives. Adv. Chem. Eng. 2001, 28, 21-79.

(17) Adam, G.; Gibbs, J. H. On the Temperature Dependence of Cooperative Relaxation Properties in Glass-Forming Liquids. J. Chem. Phys. 1965, 43, 139-146.

(18) Angell, C. A. Formation of Glasses from Liquids and Biopolymers. Science 1995, 267, 1924-1935.

(19) Ediger, M. D.; C., A.; Nagel, S. R. Supercooled Liquids and Glasses. J. Phys. Chem. 1996, 100, 13200-13212.

(20) Paluch, M.; Wojnarowska, Z.; Hensel-Bielowka, S. Heterogeneous Dynamics of Prototypical Ionic Glass CKN Monitored by Physical Aging. Phys. Rev. Lett. 2013, 110, 015702.

(21) Dingwell, D. B.; Webb, S. L. Relaxation in Silicate Melts. Eur. J. Mineral. 1990, 2, 427-449.

(22) Bohmer, R.; Ngai, K. L.; Angell, C. A.; Plazek, D. J. Nonexponential Relaxations in Strong and Fragile Glass Formers. J. Chem. Phys. 1993, 99, 4201-4209.

(23) Haller, W.; Blackburn, D. H.; Simmons, J. H. Immiscibility Dome in $\left(\mathrm{Na}_{2} \mathrm{O}\right)_{x}\left(\mathrm{SiO}_{2}\right)_{1-x}$. J. Am. Ceram. Soc. 1974, 57, 120-126.

(24) Sandercock, J. In Light Scattering in Solids III; Cardona, M., Guntherodt, G., Eds.; Topics in Applied Physics; Springer: Berlin/ Heidelberg, 1982; Vol. 51; pp 173-206.
(25) Vacher, R.; Courtens, E.; Foret, M. Anharmonic versus Relaxational Sound Damping in Glasses. II. Vitreous Silica. Phys. Rev. B 2005, 72, 214205.

(26) Baldi, G.; Fontana, A.; Monaco, G.; Orsingher, L.; Rols, S.; Rossi, F.; Ruta, B. Connection between Boson Peak and Elastic Properties in Silicate Glasses. Phys. Rev. Lett. 2009, 102, 195502.

(27) Guimbretière, G.; Rufflé, B.; Vacher, R. Acoustic Damping and Dispersion in Vitreous Germanium Oxide. Phys. Rev. B 2012, 86, 094304.

(28) Malfait, W. J.; Halter, W. E. Structural Relaxation in Silicate Glasses and Melts: High-Temperature Raman Spectroscopy. Phys. Rev. B 2008, 77, 014201.

(29) Dutreilh-Colas, M.; Canizares, A.; Blin, A.; Ory, S.; Simon, P. InSitu Raman Diagnostic of Structural Relaxation Times of Silica Glasses. J. Am. Ceram. Soc. 2011, 94, 2087-2091.

(30) McMillan, P. Structural Studies of Silicate Glasses and Melts: Applications and Limitations of Raman Spectroscopy. Am. Mineral. 1984, 69, 622-644.

(31) Mysen, B.; Frantz, J. Raman Spectroscopy of Silicate Melts at Magmatic Temperatures: $\mathrm{Na}_{2} \mathrm{O}-\mathrm{SiO}_{2}, \mathrm{~K}_{2} \mathrm{O}-\mathrm{SiO}_{2}$ and $\mathrm{Li}_{2} \mathrm{O}-\mathrm{SiO}_{2}$ Binary Compositions in the Temperature Range $25-1475{ }^{\circ} \mathrm{C}$. Chem. Geol. 1992, 96, 321-332.

(32) Mysen, B.; Virgo, D.; Seifert, F. A. The Structure of Silicate Melts: Implications for Chemical and Physical Properties of Natural Magma. Rev. Geophys. 1982, 20, 353-383.

(33) Mysen, B. O.; Frantz, J. D. Silicate Melts at Magmatic Temperatures: In-Situ Structure Determination to $1651^{\circ} \mathrm{C}$ and Effect of Temperature and Bulk Composition on the Mixing Behavior of Structural Units. Contrib. Mineral. Petrol. 1994, 117, 1-14.

(34) McMillan, P. F.; Wolf, G. H.; Poe, B. T. Vibrational Spectroscopy of Silicate Liquids and Glasses. Chem. Geol. 1992, 96, 351-366.

(35) Stebbins, J.; McMillan, P.; Dingwell, D. B. In Structure, dynamics and properties of silicate melts; Ribbe, P. H., Ed.; Reviews in Mineralogy; Mineralogical Society of America: 1995; Vol. 32.

(36) Brawer, S. A.; White, W. B. Raman Spectroscopic Investigation of the Structure of Silicate Glasses. I. The Binary Alkali Silicates. J. Chem. Phys. 1975, 63, 2421-2432.

(37) Furukawa, T.; Fox, K. E.; White, W. B. Raman Spectroscopic Investigation of the Structure of Silicate Glasses. III. Raman Intensities and Structural Units in Sodium Silicate Glasses. J. Chem. Phys. 1981, $75,3226$.

(38) Lasaga, A. C. Optimization of CNDO for Molecular Orbital Calculation on Silicates. Phys. Chem. Miner. 1982, 46, 36-46.

(39) Mysen, B. O. Effect of Pressure, Temperature, and Bulk Composition on the Structure and Species Distribution in Depolymerized Alkali Aluminosilicate Melts and Quenched Melts. J. Geophys. Res.' 1990, 95, 15733-15.

(40) Mysen, B. O. Role of $\mathrm{Al}$ in Depolymerized, Peralkaline Aluminosilicate Melts in the Systems $\mathrm{Li}_{2} \mathrm{O}-\mathrm{Al}_{2} \mathrm{O}_{3}-\mathrm{SiO}_{2}, \mathrm{Na}_{2} \mathrm{O}-$ $\mathrm{Al}_{2} \mathrm{O}_{3}-\mathrm{SiO}_{2}$, and $\mathrm{K}_{2} \mathrm{O}-\mathrm{AI}_{2} \mathrm{O}_{3}-\mathrm{SiO}_{2}$. Am. Mineral. 1990, 75, 120 .

(41) Fukumi, K.; Hayakawa, J.; Komiyama, T. Intensity of Raman Band in Silicate Glasses. J. Non-Cryst. Solids 1990, 119, 297-302.

(42) Seddon, E.; TiPPett, E. J.; Turner, W. E. S. Electrical Conductivity of Sodium-Metasilicate Glasses. J. Soc. Glass Technol. 1932, 16, 450-477.

(43) Babcock, C. L. Viscosity and Electrical Conductivity of Molten Glasses. J. Am. Ceram. Soc. 1934, 17, 329-342.

(44) Tomozawa, M.; Koike, A.; Ryu, S. Exponential Structural Relaxation of a High Purity Silica Glass. J. Non-Cryst. Solids 2008, 354, $4685-4690$.

(45) Johari, G.; Goldstein, M. Viscous Liquids and the Glass Transition. II. Secondary Relaxations in Glasses of Rigid Molecules. J. Chem. Phys. 1970, 53, 2372-2388.

(46) Johari, G. Localized Molecular Motions of $\beta$-Relaxation and Its Energy Landscape. J. Non-Cryst. Solids 2002, 307, 317-325.

(47) Ngai, K.; Wright, G. Relaxations in complex systems; Naval Research Laboratory: 1985. 
(48) Blochowicz, T.; Tschirwitz, C.; Benkhof, S.; Rössler, E. A. Susceptibility Functions for Slow Relaxation Processes in Supercooled Liquids and the Search for Universal Relaxation Patterns. J. Chem. Phys. 2003, 118, 7544.

(49) Lunkenheimer, P.; Pimenov, A.; Dressel, M.; Goncharov, Y. G.; Böhmer, R.; Loidl, A. Fast Dynamics of Glass-Forming Glycerol Studied by Dielectric Spectroscopy. Phys. Rev. Lett. 1996, 77, 318-321.

(50) Tanaka, H. Origin of the Excess Wing and Slow Beta Relaxation of Glass Formers: A Unified Picture of Local Orientational Fluctuations. Phys. Rev. E 2004, 69, 021502.

(51) Tanaka, H. Two-Order-Parameter Description of Liquids. II. Criteria for Vitrification and Predictions of Our Model. J. Chem. Phys. 1999, 11, 3175.

(52) Hu, L.; Yue, Y. Secondary Relaxation Behavior in a Strong Glass. J. Phys. Chem. B 2008, 112, 9053-9057.

(53) Poole, J. P. Viscosité à Basse Température des Verres AlcalinoSilicates. Verres Réfract. 1948, 2, 222-228.

(54) Dingwell, D. B.; Webb, S. L. Structural Relaxation in Silicate Melts and Non-Newtonian Melt Rheology in Geologic Processes. Phys. Chem. Mineral. 1989, 16, 508-516.

(55) Angell, C. A.; Cheeseman, P. A.; Tamaddon, S. J. Phys. Colloq. 1981, 43, C9-381.

(56) Greaves, G. EXAFS and the Structure of Glass. J. Non-Cryst. Solids 1985, 71, 203-217.

(57) Greaves, G. N.; Fontaine, A.; Lagarde, P.; Raoux, D.; Gurman, S. J. Local Structure of Silicate Glasses. Nature 1981, 293, 611-616.

(58) Farnan, I.; Grandinetti, P. J.; Baltisberger, J. H.; Stebbins, J. F.; Werner, U.; Eastman, M. A.; Pines, A. Quantification of the Disorder in Network-Modified Silicate Glasses. Nature 1992, 358, 31-35.

(59) Smith, W.; Greaves, G. N.; Gillan, M. J. Computer Simulation of Sodium Disilicate Glass. J. Chem. Phys. 1995, 103, 3091-3097.

(60) Chengde, H.; Cormack, A. N. Structural Differences and Phase Separation in Alkali Silicate Glasses. J. Chem. Phys. 1991, 95, 36343642.

(61) Greaves, G. N.; Ngai, K. L. Reconciling Ionic-Transport Properties with Atomic Structure in Oxide Glasses. Phys. Rev. B 1995, 52, 6358-6380.

(62) Jund, P.; Kob, W.; Jullien, R. Channel Diffusion of Sodium in a Silicate Glass. Phys. Rev. B 2001, 64, 134303.

(63) Meyer, A.; Horbach, J.; Kob, W.; Kargl, F.; Schober, H. Channel Formation and Intermediate Range Order in Sodium Silicate Melts and Glasses. Phys. Rev. Lett. 2004, 93, 27801.

(64) Meneses, D. M.; Eckes, M.; Del Campo, L.; Cristiane, N. S.; Vaills, Y.; Echegut, P. Investigation of Medium Range Order in Silicate Glasses by Infrared Spectroscopy. Vib. Spectrosc. 2013, 65, 50-57.

(65) Vassiliy, L.; Peter, G. W. Theory of Aging in Structural Glasses. J. Chem. Phys. 2004, 121, 2852-2865.

(66) Ranko, R. Heat Capacity in the Glass Transition Range Modeled on the Basis of Heterogeneous Dynamics. J. Chem. Phys. 2011, 134, 144501. 